\title{
MiR-137 Suppresses Triple-Negative Breast Cancer Stemness and Tumorigenesis by Perturbing BCL11A-DNMT1 Interaction
}

\author{
Feiyu Chen Na Luo Yu Hu Xin Li Kejing Zhang \\ Department of Breast Surgery, Xiangya Hospital, Central South University, Changsha, China
}

\section{Key Words}

Mir-137 • B-cell lymphoma/leukemia 11A (BCL11A) - Triple negative breast cancer • Breast cancer stem cells $\bullet$ DNMT1

\begin{abstract}
Background/Aims: Triple negative breast cancer (TNBC) is resistant to conventional chemotherapy due to high proportions of cancer stem cells (CSCs). The aim of this study is to unravel the miR-137-mediated regulatory mechanism of B-cell lymphoma/leukemia $11 \mathrm{~A}$ (BCL11A) in TNBC. Methods: A corhort of 34 TNBC tumor tissues and paired adjacent normal tissues, as well as 25 non-TNBC tumor tissues and paired adjacent normal tissues were collected post-operatively from patients with breast cancer. Q-PCR was performed to determine the mRNA levels of miR-137 and BCL11A in breast tissues and cell lines. Bioinformatics analysis and dual luciferase reporter assay were used to verify the direct interaction between miR-137 and $B C L 11 A$. After up-/down-regulation of BCL11A, miR-137, or DNMT1 via lentiviral transduction in TNBC cell lines SUM149 and MDA-MB-231 cells, Q-PCR and Western blot assays were used to detect the expression levels of BCL11A, DNA methyltransferases 1 (DNMT1), and Islet-1 (ISL1). Mammosphere assay was conducted to assess tumorosphere formation ability of cells, coupled with flow cytometry to determine the percentage of breast cancer stem cells. Co-immunoprecipitation assay was used to determine the interaction between BCL11A and DNMT1. Xenograft tumorigenesis assay was performed to monitor tumor formation in vivo. Results: $B C L 11 \mathrm{~A}$ was highly expressed in TNBC, whereas miR-137 was significantly lower in both TNBC tissues and cell lines. miR-137 suppressed BCL11A expression at both mRNA and protein levels by directly targeting its 3'UTR. In both SUM149 and MDA-MB-231 cells, overexpression of miR-137 or knockdown of BCL11A reduced the number of tumoroshperes and the percentage of cancer stem cells in vitro, and inhibited tumor development in vivo. Furthermore, BCL11A interacted with DNMT1 in TNBC cells. Silencing of either BCL11A or DNMT1 impaired cancer stemness and tumorigenesis of TNBC via suppressing ISL1 expression both in vitro, and in vivo. Conclusions: By perturbing BCL11A-DNMT1 interaction, miR-137 impairs cancer stemness and suppresses tumor development in TNBC.




\section{Cellular Physiology Cell Physiol Biochem 2018;47:2147-2158 \\ \begin{tabular}{l|l} 
DOI: 10.1159/000491526 & $\begin{array}{l}\text { O 2018 The Author(s). Published by S. Karger AG, Basel } \\
\text { www.karger.com/cpb }\end{array}$
\end{tabular} \\ Chen et al.: MiR-137 Impairs Cancer Stemness and Tumorigenesis in TNBC}

\section{Introduction}

Breast cancer is the most common cancer in women worldwide, and it is highly heterogeneous. In order to refine the clinical decision, breast cancer has been classified into several subtypes based on the immunohistochemical characterization of estrogen receptor (ER), progesterone receptor (PR) and human epidermal growth factor receptor type 2 (HER2) expression including luminal A subtype, luminal B subtype, HER2-positive subtype, and triple negative type [1,2]. The triple negative breast cancer (TNBC) accounts for $12 \sim 17 \%$ of breast cancer in women, which is characterized by a lack of ER, PR and HER2 expression $[3,4]$. Moreover, the percentage of $\mathrm{CD}_{4} 4^{+} / \mathrm{CD} 24^{-/ \text {low }}$ cancer stem cells (CSC) is higher in TNBC than that in other subtypes of breast cancer $[3,5]$. Conventional chemotherapy remains the most common therapeutic option for TNBC, since patients with TNBC could not be treated with endocrine therapy or drugs that target HER2[6]. However, chemotherapy resistance due to the high percentage of CD44 $/ \mathrm{CD} 24^{-/ \text {low }} \mathrm{CSCs}$ resulting in a poor prognosis of TNBC[7], . Therefore, it is important to develop a novel targeted therapy to improve the clinical outcome of TNBC.

MicroRNAs (miRNAs) are non-coding single-stranded RNA molecules which contain $\sim 22$ nucleotides, which function as post-transcriptional regulators of target genes. MiRNAs play important roles in diverse biological processes [8]. Accumulating evidence suggests that miRNAs are involved in cancer development and progression, serving as either oncogenes or tumor suppressors [9, 10]. Recent studies have demonstrated that miR-137 is down-regulated in several types of cancers including non-small-cell lung cancer (NSCLC), colorectal cancer and glioblastoma, indicating that miR-137 might act as a tumor suppressor in cancer cells [11-13]. More important, miR-137 negatively regulates the orphan nuclear receptor estrogen-related receptor $\alpha(E R R \alpha)$, and impairs cell proliferation and migration in breast cancer cells [14]. It has also been reported that miR-137 suppresses cell invasion and epithelial-mesenchymal transition (EMT) by targeting carboxyl-terminal binding protein 1 (CtBP1) in non-TNBC cell line MCF7 cells [15]. More recently, it has been reported that miR137 is highly expressed in the developing mammary gland, inducing gland formation and invasion [16]. Furthermore, overexpression of miR-137 impairs cell proliferation, migration, tumor formation and EMT in TNBC cell line MDA-MB-231 cells $[14,16]$. However, the underlying regulatory mechanism involving miR-137 in TNBC remains uninvestigated.

The B-cell lymphoma/leukemia 11A (BCL11A) gene encodes a zinc-finger transcription factor, which plays a critical role in hemoglobin switching [17]. It also serves as an oncogene in B-cell malignancies $[18,19]$, but BCL11A involvement in solid tumors has been rarely reported until recent years. Khaled et al. revealed a novel tumorigenic function of BCL11A in TNBC. In brief, BCL11A is highly expressed in TNBC, and exogenous BCL11A overexpression in TNBC promotes tumor development in vitro and in vivo [20]. These findings indicate that BCL11A might be a potential candidate for targeted therapy in TNBC. However, the exact mechanism is still unknown.

DNA methyltransferases 1 (DNMT1), a member of DNA methyltransferases family, is responsible for DNA methylation at cytosine-phosphoguanine (CpG) islands upstream of tumor suppressor genes [21]. Previous studies have demonstrated that DNMT1 is required for maintenance of CSC in a variety of cancers, including prostate cancer, pancreatic cancer and breast cancer [22-24]. For instance, DNMT1 induces histone demethylation of H3K9me3 and H3K27me3 on the Zeb2 and KLF4 promoter in prostate cancer cells [23]. More important, a recent study has demonstrated that DNMT1 is up-regulated in mammary tumors. DNMT1 inhibition or DNMT1-induced hypermethylation/down-regulation of Islet-1 (ISL1) limits CSC population in breast cancer cells [22]. On the other hand, a proteomic screen has shown that DNMT1 is a BCL11A-accociated protein in erythroid cells, and it is required for maintenance of fetal hemoglobin ( $\mathrm{HbF}$ ) silencing in vitro and in vivo [25], indicating that DNMT1 co-works with BCL11A to modulate HbF silencing in eythroid cells. In addition, disruption of STAT3-DNMT1 interaction causes re-expression of tumor suppressor genes through de-methylation and inhibits tumor growth in TNBC [26], suggesting that the 
interaction between transcription factor(s) and DNMT1 might be crucial for tumorigenesis in TNBC. These observations thus lead us to speculate that BCL11A/DNMT1 plays an important role in regulating cancer stemness and tumorigenesis in TNBC.

In this study, we predicted that miR-137 targeted 3' UTR of BCL11A using bioinformatics analysis. Thus we hypothesize that miR-137 likely serves as a tumor suppressor of TNBC, which could suppressed cancer stemness and tumor development of TNBC by silencing BCL11A, and remove the inhibition of ISL1 due to BCL11A-DNMT1 interaction.

\section{Materials and Methods}

Isolation of human normal and breast tumor tissues

A corhort of 34 TNBC tumor tissues and paired adjacent normal tissues, as well as 25 non-TNBC tumor tissues and paired adjacent normal tissues were collected post-operatively from patients with breast cancer in Xiangya Hospital, Central South University. Consents from all patients were obtained. Diagnoses of breast cancer were given by two pathologists. All patients recruited to this study did not receive any pre-operative treatment.

\section{Animals}

6 8-week-old female BALB/c nude mice $(n=60)$ were purchased from Hunan SJA Laboratory Animal Co., Ltd (Changsha, China). All mice were housed in a controlled environment, with room temperatures (22 ${ }^{\circ} \mathrm{C}$ ) and light/darkness cycles of $12 \mathrm{~h}$, for at least $7 \mathrm{~d}$ before the experiments. All experiments were approved by the Animal Ethical Committee, Xiangya School of Medicine, Central South University, and performed following the guidelines of the Animal Care and Use Committee of Xiangya School of Medicine, Central South University.

\section{Cell culture and transduction of lentivirus}

Human mammary epithelial cell line MCF10A (Cat.no. CBP60419), human non-TNBC cell line MCF7 (ER/PR +ve/HER-2 -ve, Cat. no. TCHu74), human TNBC cell line MDA-MB-231 (Cat. no. TCHu227), and human embryonic kidney (HEK)293 cells (Cat.no.GNHu43) were obtained from Cell Bank of Type Culture Collection, Chinese Academy of Science (Shanghai, China). Human TNBC cell line. SUM149 was given as a gift by Professor Zhimin Shao from Department of Breast Surgery, Fudan University Shanghai Cancer Center. MCF10A cells were grown in F12/DMEM supplemented with 5\% FBS, $10 \mu \mathrm{g} / \mathrm{ml}$ insulin, $20 \mathrm{ng} / \mathrm{ml}$ epidermal growth factor (EGF), $100 \mathrm{ng} / \mathrm{ml}$ cholera toxin, $0.5 \mu \mathrm{g} / \mathrm{ml}$ hydrocortisone (Sigma-Aldrich, St Louis, MO, USA). MCF7, MDA-MB-231 and HEK293T cells were cultured in DMEM containing 10\% FBS and 100 units/ml penicillin/streptomycin. SUM149 cells were grown in Ham's F12 medium supplemented with 5\% FBS, 1 ug/ $\mathrm{ml}$ hydrocortisone and $5 \mu \mathrm{g} / \mathrm{ml}$ insulin. Cultures were maintained at $37^{\circ} \mathrm{C}$ in humidified atmosphere with $5 \% \mathrm{CO}_{2}$ in air.

SUM149 and MDA-MB-231 cells were transduced with the lentivirus that either overexpressing miR137, BCL11A, DNMT1, or their short hairpin RNA (shRNA), respectively (Feng Hui Biotechnology Co., Ltd, Changsha, Hunan, China). After antibiotic selection of puromycin for 2-3 weeks, the stable clones were obtained.

\section{Dual luciferase reporter assays}

Wild-type 3' UTR of BCL11A were amplified by PCR. The mutant BCL11A 3' UTR was synthesized using QuikChange Lightning Multi Site-Directed Mutagenesis Kit (Agilent Technologies, Palo Alto, CA, USA). Both plasmids were then cloned downstream of Renilla luciferase gene in the psiCHECK $^{\mathrm{TM}}-2$ vector (Promega, Madison, WI, USA), and purified using commercial plasmid purification kit (Qiagen, Valencia, CA, USA), and finally co-transfected with either scrambled miRNA or miR-137 mimics into HEK293 cells using FuGENE ${ }^{\mathbb{H}} \mathrm{H}$ transfection reagent (Promega) . The dual luciferase reporter assay was performed $48 \mathrm{~h}$ after transfection using dual luciferase assay system (Promega) on a GLOMAX ${ }^{\mathrm{TM}}$ 20/20 Luminometer (Promega). The luciferase activity of each sample was normalized to the firefly luciferase activity. All experiments were performed in triplicate. 


\section{Cellular Physiology Cell Physiol Biochem 2018;47:2147-2158 \begin{tabular}{l|l} 
and Biochemistry Published online: July 04, 2018 & $\begin{array}{l}\text { (c) } 2018 \text { The Author(s). Published by S. Karger AG, Basel } \\
\text { www.karger.com/cpb }\end{array}$
\end{tabular}}

\section{MTT assay}

Cells were seeded into 96-well plates and incubated for $24 \mathrm{~h}$ prior to MTT assay. $20 \mu \mathrm{l}$ MTT was added into each well at different time points, and incubated for $4 \mathrm{~h}$ at $37^{\circ} \mathrm{C}$. Culture medium was then removed and MTT formazan crystals were resolved in $150 \mu \mathrm{l}$ DMSO (Sigma-Aldrich). Absorbance values were measured at a wavelength of $570 \mathrm{~nm}$ by the use of microplate reader (Model 680, Bio-Rad Laboratories, Hercules, CA, USA). All procedures were repeated at least 3 times.

\section{Mammosphere assays}

Mammosphere assays were performed as previously described [27]. In brief, cells were cultured in suspension in serum-free DMEM/F12 medium supplemented with B27 (Gibco, Grand Island, NY, USA) and $20 \mathrm{ng} / \mathrm{ml} \mathrm{EGF,} \mathrm{0.4 \%} \mathrm{BSA} \mathrm{(Sigma-Aldrich)} \mathrm{and} 4 \mathrm{mg} / \mathrm{ml}$ insulin. 21 days later, the mammosphere were photographed and counted.

\section{Identification and isolation of CSC via flow cytometry (FACS)}

FACS was performed as previously described with minor modifications [28]. CD24 and CD44 expression were analyzed in 21-day-old mammospheres following incubation in trypsin-EDTA. Cells were pelleted by centrifugation at $4{ }^{\circ} \mathrm{C}$, resuspended with PBS. and stained with anti-CD24-FITC and anti-CD44-PE antibody (eBioscience, San Diego, CA, USA) for $20 \mathrm{~min}$ at $4{ }^{\circ} \mathrm{C}$. For CSC isolation, cells were sorted on a FACScalibur flow cytometer using BD FACS Diva Software (BD Biosciences, San Jose, CA, USA). Side scatter and forward scatter profiles were used to eliminate debris and cell doublets.

\section{RNA isolation and Quantitative-PCR ( $Q-P C R$ )}

Total RNA was isolated from normal/tumor tissues of patients with TNBC or different cell lines using TRIzol reagent (Thermo Fisher Scientific, Waltham, MA) and reverse transcribed with Advantage RT-for-PCR Kit (Takara, Japan). cDNA products were used as templates for Q-PCR with specific primers. The following primers were used in this study: miR-137, forward 5'-GCG CGC TTA TTG CTT AAG AAT AC-3' and reverse 5'-GTC GTA TCC AGT GCA GGG TCC GAG GTA TTC GCA CTG GAT ACG ACC TAC GC-3'; BCL11A, forward 5'-ATG CGA GCT GTG CAA CTA TG-3' and reverse 5'-CAA CAC TCG ATC ACT GTG CC-3'; DNMT1, forward 5'-CCG ACT ACA TCA AAG GCA GC-3' and reverse 5'-AGG TTG ATG TCT GCG TGG TA-3'; ISL-1, forward 5'-AAA CAG GAG CTC CAG CAA AA-3' and reverse 5'-GCT ACA GGA CAG GCC AAG AG-3'; GAPDH, forward 5'-CCA GGT GGT CTC CTC TGA-3' and reverse 5'-GCT GTA GCC AAA TCG TTG T-3'; U6, forward 5'-CTC GCT TCG GCA GCA CA3' and reverse 5'-AAC GCT TCA CGA ATT TGC GT-3'. The mRNA levels of the target genes were analyzed by SYBR green method with iQTM SYBR $®$ Green Supermix reagent (Bio-Rad Laboratories, Hercules, CA, USA) according to the manufacturer's instructions and determined using $2{ }^{-\Delta \Delta C}$ method ( $n=3$, each in triplicate). The GAPDH or U6 was used as an internal control for normalization. The specificity of the fluorescence signal was confirmed by both melting curve analysis and agarose gel electrophoresis.

\section{Western Blot}

Protein lysate from MDA-MB-231 and SUM149 cells were prepared with RIPA lysis buffer supplemented with fresh protease and phosphatase inhibitor Cocktail (Sigma-Aldrich). Total protein concentration was determined by Bradford assay (Bio-Rad). Equal amount of protein lysate (50ug) were resolved in SDS-PAGE, transferred to PVDF membranes (Bio-Rad). Membranes were blocked with 5\% non-fat milk powder [w/v] in PBS/Tris/0.1\% Tween-20 for $1 \mathrm{~h}$, followed by incubation with primary (1:800-1000) and respective secondary antibodies (1:10000). Primary antibodies against BCL11A (ab19487), DNMT1 (ab19905), ISL-1 (ab109517), and $\beta$-actin (ab8226) were obtained from Abcam (Cambridge, MA, USA). After incubation and wash with TBST, membranes were visualized using ECL western blotting detection system.

\section{Co-Immunoprecipitation (Co-IP)}

Co-IP was performed as described [29]. In brief, cell lysates were prepared with IP lysis buffer (50 mM Tris- $\mathrm{HCl}, 150 \mathrm{mM} \mathrm{NaCl}, 10 \%$ NP-40, $1 \mathrm{mM}$ EDTA, 10\% glycerol, $10 \mathrm{mM} \mathrm{NaF}, 1 \mathrm{mM} \mathrm{Na} \mathrm{VO}_{4}, 1 \mathrm{mM}$ DTT, $1 \mathrm{mM}$ PMSF and protease inhibitor cocktail). Protein lysates were incubated with specific antibody at $4{ }^{\circ} \mathrm{C}$ overnight, followed by incubation with Protein A-Sepharose CL-4B beads (Pharmacia, Uppsala, Sweden). Immnocomplexes were collected and washed 4 times, then subjected to western blotting analysis. 


\section{Cellular Physiology Cell Physiol Biochem 2018;47:2147-2158

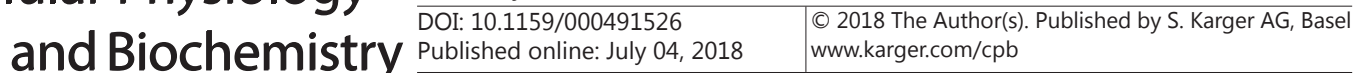

Chen et al.: MiR-137 Impairs Cancer Stemness and Tumorigenesis in TNBC

\section{Xenograft tumorigenesis assay}

1.0x10 ${ }^{6}$ MDA-MB-231 and SUM149 cells were suspended in 25\% Matrigel (BD Biosciences) and HBSS, and injected subcutaneously into the flank of BALB/c nude mice of 6-8-week age. Tumor sizes were measured and tumor volumns were calculated. Xenograft mice were sacrificed 4 weeks after the injection, and tumors were dissected for analysis.

\section{Stataistical analysis}

All experiments were performed for at least three times. Data are expressed as the means \pm S.D. Statistical analysis was performed by two-way ANOVA. In selected experiments, a Student's t-test was used for paired comparisons. Statistical analysis was performed using SPSS 21.0. Significance was set at ${ }^{*}, \mathrm{P}<$ $0.05 ; * *, \mathrm{P}<0.01$.

\section{Results}

miR-137 is down-regulated and BCL11A is up-regulated in triple negative breast cancer tissues and cell lines

In order to determine the expression levels of miR-137 and BCL11A, Q-PCR was performed with TNBC tissues, non-TNBC tissues, as well as their paired adjacent normal breast tissues. As shown in Fig. 1A, miR-137 was significantly lower in TNBC tissues and nonTNBC tumor tissues compared with that in paired adjacent normal breast tissues, respectively. BCL11A was highly expressed in TNBC tissues when compared with paired adjacent normal breast tissues. Whereas there was no significant difference in the expression level of BCL11A between non-TNBC tumor tissues and paired adjacent normal breast tissues (Fig. 1B).

Next we examined the expression levels of miR-137 and BCL11A in 4 different mammary cell lines. Similarly, the expression levels of miR-137 were down-regulated in all three breast cancer cell lines, compared with normal mammary epithelial cell line MCF10A (Fig. 1C). A 1.52-fold and 2.73-fold increase in the expression levels of BCL11A were observed in TNBC cell lines, MDA-MB-231 cells and SUM149 cells, respectively, compared with that in MCF10A cells. However, BCL11A expression in MCF7 cells (a non-TNBC cell line) showed no significant difference when compared with MCF10A cells (Fig. 1D). Taken together, miR-137 is significantly down-regulated in both triple-negative and non-triple negative breast cancer tissues and cells, whereas BCL11A is exclusively up-regulated in TNBC tissues and cells.

Fig. 1. Expression of BCL11A and miR-137 in breast tumors and cell lines. A) mRNA levels of miR-137 in TNBC, non-TNBC and paired adjacent normal breast tissues were determined by Q-PCR. U6 served as an internal control. Each bar is a mean \pm SD of $n=$ 3 experiments. * indicates $\mathrm{P}<0.05$. B) mRNA levels of BCL11A in TNBC, non-TNBC and paired adjacent normal breast tissues were determined by Q-PCR. GAPDH served as an internal control. Each bar is a mean \pm SD of $\mathrm{n}=3$ experiments. * indicates $\mathrm{P}<0.05$. C) mRNA levels of miR-137 in different mammary cell lines were determined by Q-PCR. U6 served as an internal control. Each bar is a mean \pm SD of $n$ $=3$ experiments. ${ }^{*}$ indicates $\mathrm{P}<0.05$, ** indicates $\mathrm{P}<0.01$. D) mRNA levels of BCL11A in different mammary cell lines were determined by Q-PCR. GAPDH served as an internal control. Each bar is a mean \pm SD of $\mathrm{n}=3$ experiments. * indicates $\mathrm{P}<0.05$, ** indicates $\mathrm{P}<0.01$.

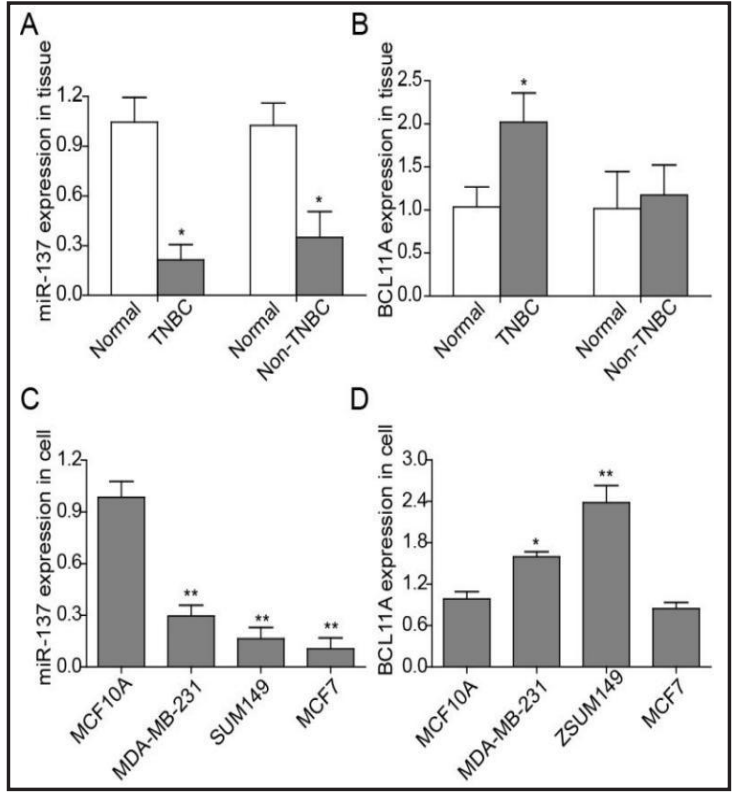




\section{Cellular Physiology Cell Physiol Biochem 2018;47:2147-2158 \begin{tabular}{l|l|l} 
DOI: 10.1159/000491526 & $\begin{array}{l}\text { O } 2018 \text { The Author(s). Published by S. Karger AG, Basel } \\
\text { www.karger.com/cpb }\end{array}$
\end{tabular}

miR-137 down-regulates BCL11A expression by directly targeting its 3'UTR

To check whether there is a correlation between the high expression of BCL11A and the low expression of miR-137 in TNBC, a set of online bioinformatics softwares, namely TargetScan (http://www.targetscan.org/) and miRanda (http://www.microrna.org/ microrna/searchMirnas.do), were used to analyze and predict the binding site of miR-137 in 3' UTR of BCL11A gene (Fig. 2A) . In addition, as shown in Fig. 2B, miR-137 mimics decreased BCL11A mRNA level by 70\% in SUM149 cells, and 55\% in MDA-MB-231 cells, respectively. Similar effects were also found at the protein level by western blot (Fig. 2B).

To further verify the regulatory effect of miR-137 on BCL11A, wild-type and mutant 3' UTR of BCL11A were cloned into psiCHECK ${ }^{\mathrm{TM}}-2$ vector, respectively (Fig. 2C). Dual luciferase reporter assays showed that co-transfection of wild-type BCL11A reporter (BCL11A) and miR-137 caused a significant reduction of luciferase activity compared with co-transfection of BCL11A and scrambled miRNA (Fig. 2D). In contrast, mutant BCL11A reporter (mut11A)

Fig. 2. MiR-137 reduces BCL11A expression by directly targeting its 3' UTR. A) The results of TargetScan and MiRanda predicted that miR137 targets 3' UTR of BCL11A. B) BCL11A mRNA and protein levels were determined by Q-PCR and western blot. GAPDH served as an internal control for Q-PCR. $\beta$-actin served as a loading control for western blot. Each bar is a mean \pm SD of $\mathrm{n}=3$ experiments. * indicates $\mathrm{P}<0.05$. $\mathrm{C}$ ) The sequence of wild-type or mutated BCL11A

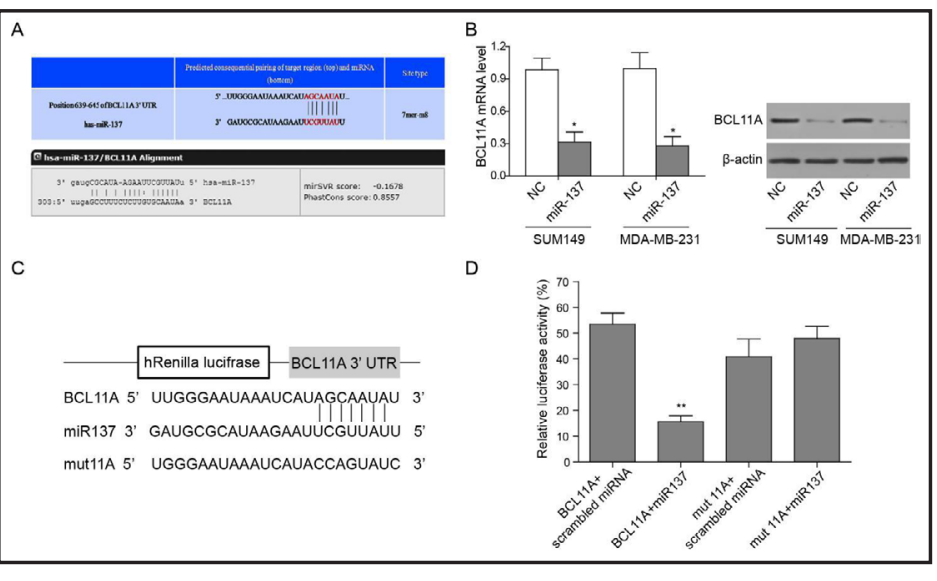
3' UTR. D) SUM149 and MDA-

MB-231 cells were co-transfected with BCL11A/mut11A and scrambled miRNA/miR-137. Dual luciferase reporter assay was performed to measure the luciferase activity (BCL11A=wild-type BCL11A reporter; mut11A=mutant BCL11A reporter). Each bar is a mean \pm SD of $n=3$ experiments. * indicates $\mathrm{P}<0.05, * *$ indicates $\mathrm{P}<0.01$.

Fig. 3. MiR-137 inhibits cancer stemness of TNBC by downregulating BCL11A in vitro. A) Cell proliferation was monitored by MTT assay. Data were presented as mean $\pm \mathrm{SD}$ in triplicate. B) Comparison of the number of suspension mammospheres formed from control cells, miR137 overexpressed cells and BCL11A knockdown cells, both in SUM149 and MDA-MB-231 cells. Each bar is a mean \pm SD of $n=3$ experiments. $*$ indicates $\mathrm{P}<0.05$. C) Percentage of $\mathrm{CD} 44^{+} / \mathrm{CD} 24^{-} /$ low cells in the mammospheres of SUM149 and MDA-MB-231 cells were detected by flow cytometry.

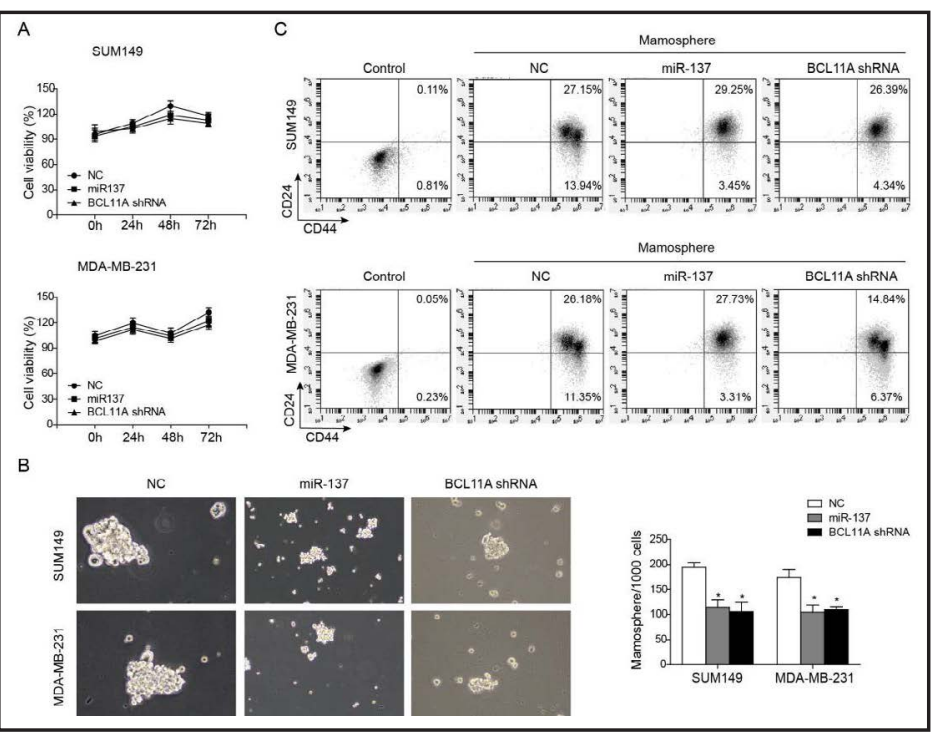
Data are representative images of 3 independent experiments.

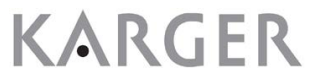


with mismatch sequences inserted in the potential binding site for miR-137 successfully abolished the repression effect of miR-137 on luciferase activity (Fig. 2D). Collectively, these data indicate that miR-137 negatively regulates BCL11A expression through directly binding to its 3'UTR.

\section{MiR-137 inhibits cancer stemness of TNBC by down-regulating BCL11A in vitro}

Previous study suggests that BCL11A acts as an oncogene in TNBC [20]. We next examined whether miR-137-mediated down-regulation of BCL11A would inhibit tumor development in TNBC cells. Overexpression of miR-137 or knockdown of BCL11A had no significant effects on cell proliferation in both SUM149 and MDA-MB-231 cells (Fig. 3A).

Breast cancer stem cells (BCSC) are tumor cells with capability of self-renewal, differentiation and metastasis. These cells persist in tumors, and are considered to be associated with poor prognosis of breast cancer [30-32]. Noting that CD44 $4^{+}$and CD24-/low are generally accepted markers of BCSC, mammosphere assay and flow cytometry were performed to analyze regulatory roles of miR-137/BCL11A in BCSCs [30-32]. As shown in Fig. 3B, overexpression of miR-137 led to a significant reduction in the number of tumorospheres in both SUM149 and MDA-MB-231 cells. Knockdown of BCL11A using specific shRNA also reduced the number of tumorospheres by $\sim 50 \%$ (Fig. 3B), suggesting that miR-137and/or shRNA-mediated down-regulation of BCL11A inhibit the formation of suspension mammosphere. In addition, flow cytometry was used to assess $C D 44^{+} / \mathrm{CD} 24^{-/ / 10 w}$ cell ratio in propagated mammospheres. Compared to the $0.81 \%$ positive expression of $\mathrm{CD} 44^{+}$/ CD24//low in SUM149 monolayer culture cells, the expression of CD44 $/$ CD24-/low in SUM149 mammosphere cells was significantly elevated to $13.94 \%$ (Fig. 3C, upper panel). Similar results were observed in MDA-MB-231 cells (Fig. 3C, lower panel). Both overexpression of miR-137 and knockdown of BCL11A remarkably reduced the BCSC ratio in both SUM149 and MDA-MB-231 mammospheres (Fig. 3C). Collectively, miR-137-mediated down-regulation of BCL11A inhibits cancer stemness and tumor development of TNBC cells.

MiR-137 inhibits tumor formation by down-regulateing BCL11A in vivo

To further verify the effects of miR-137/BCL11A on tumor formation in vivo, nude mice xenograft models were generated by subcutaneous injection of either miR-137-overexpressed or BCL11A-knockdown SUM149 and MDA-MB-231 cells. Tumors were harvested 4 weeks after tumor cell implantation. The expression levels of miR-137 and BCL11A in xenograft tumors in different groups were verified, showing a significantly elevation of miR-137 in miR-137-overexpressed model, and reduction of BCL11A in BCL11A knockdown model (Fig. $4 \mathrm{~A})$.

As shown in Fig. 4B, both normal SUM149 and MDA-MB-231 cells formed tumors in nude mice successfully, while miR-137-overexpressed or BCL11A-knockdown cells caused

Fig. 4. MiR-137 inhibits tumor formation by down-regulateing BCL11A in vivo. A) mRNA levels of miR137 and BCL11A in xenograft tumors were determined by Q-PCR. GAPDH served as an internal control. Each bar is a mean $\pm \mathrm{SD}$ of $\mathrm{n}=3$ experiments. * indicates $\mathrm{P}<0.05$, ** indicates $\mathrm{P}<0.01$. B) Effect of miR-137 overexpression and BCL11A knockdown on tumor formation in vivo. C) Quantitative analysis of tumor volumes in miR-137 overexpressed group and BCL11A

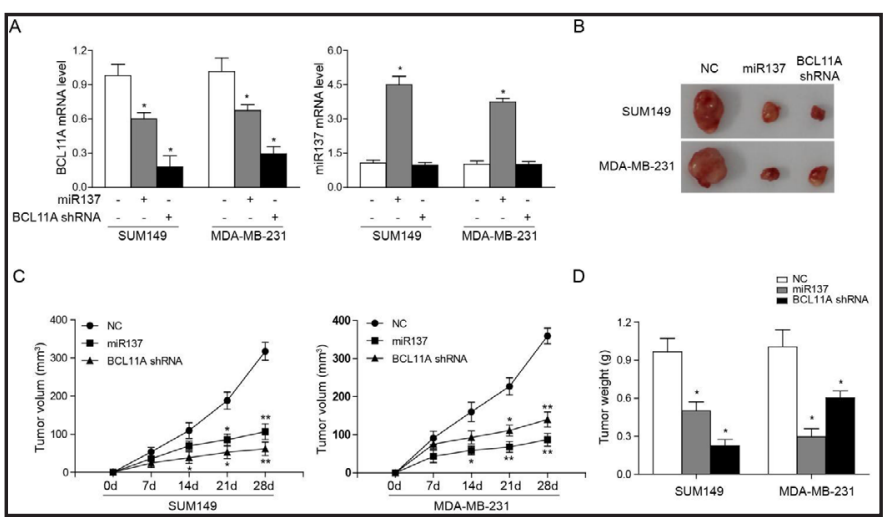
knockdown group. Each bar is a mean \pm SD of $n=3$ experiments. * indicates $\mathrm{P}<0.05$, ** indicates $\mathrm{P}<0.01$. D) Quantitative analysis of tumor weights in miR-137 overexpressed group and BCL11A knockdown group. Each bar is a mean \pm SD of $\mathrm{n}=3$ experiments. * indicates $\mathrm{P}<0.05$. 


\section{Cellular Physiology Cell Physiol Biochem 2018;47:2147-2158 \begin{tabular}{l|l} 
DOI: 10.1159/000491526 & O 2018 The Author(s). Published by S. Karger AG, Basel \\
www.karger.com/cpb
\end{tabular}

Fig. 5. BCL11A directly interacts with DNMT1 in TNBC cells. A and C) DNMT1 mRNA level was determined by Q-PCR. GAPDH served as an internal control for Q-PCR. Each bar is a mean $\pm S D$ of $n=3$ experiments. $B$ and D) DNMT1 protein level was determined by western blot. $\beta$-actin served as a loading control. Data are representative images of 3 independent experiments. E) The interaction between BCL11A and DNMT1 was determined by co-IP. Whole cell lyasates (WCL) served as control for co-IP. Data are representative images of 3 independent experiments.

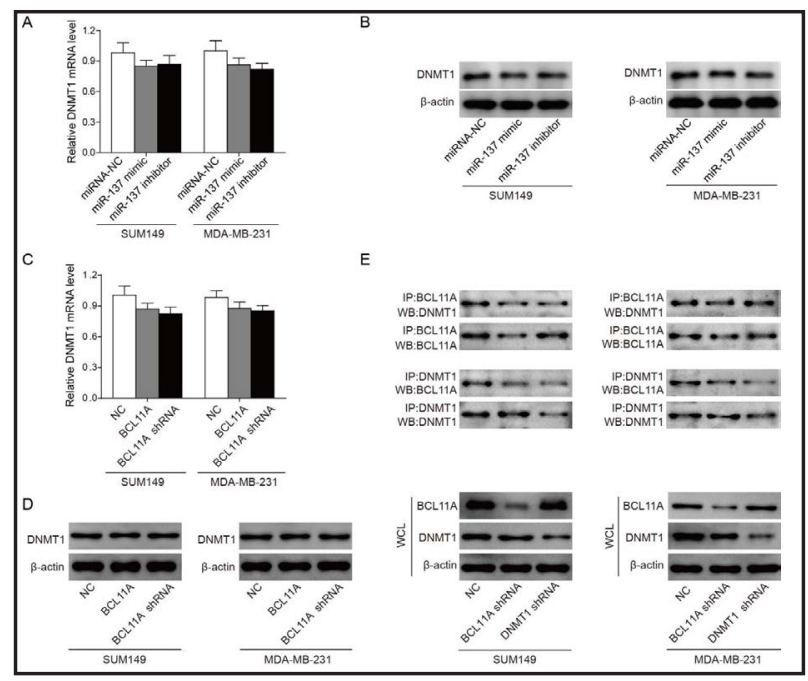

dramatic reduction in tumor sizes. Moreover, the volumes and weights of tumors were measured and showed in Fig. 4C and 4D, overexpression of miR-137 or knockdown of BCL11A resulted in reduction of both tumor volumes and weights of tumors in vivo. In summary, these findings illustrate that miR-137-mediated down-regulation of BCL11A inhibits tumor formation in xenograft models.

\section{BCL11A directly interacts with DNMT1 in TNBC cells}

Given that DNMT1 is a BCL11A-associated protein in erythroid cells [25], we investigated whether DNMT1 cooperated with BCL11A through direct interaction in TNBC cells. We first tested the effect of miR-137 or BCL11A on DNMT1 mRNA and protein levels. As shown in Fig. $5 A$ and $5 B$, transfection of miR-137 mimics or inhibitor showed few effects on either mRNA or protein levels of DNMT1 in both SUM149 and MDA-MB-231 cells. Similarly, overexpression or knockdown of BCL11A caused no significant changes in DNMT1 expression in both SUM149 and MDA-MB-231 cells (Fig. 5C and 5D). To further verify the interaction between BCL11A and DNMT1, co-IP assay was performed. Knockdown of either BCL11A or DNMT1 disrupted BCL11A-DNMT1 interaction (Fig. 5E, $1^{\text {st }}$ and $3^{\text {rd }}$ row). Meanwhile, whole cell lysates (WCL) served as the control for co-IP, illustrating that the specific shRNA of BCL11A or DNMT1 successfully knockdown the expression of its target gene in both SUM149 and MDA-MB-231 cells (Fig. 5E, lower panel). Collectively, these data indicated that instead of regulating its expression, BCL11A interacts with DNMT1 by directly binding in TNBC cells.

\section{BCL11A/DNMT1 is responsible for breast cancer stemness in vitro}

A previous study has illustrated that DNMT1 is required for CSC maintenance and tumorigenesis in breast cancer [22]. To study the effect of BCL11A and DNMT1 on cancer stemness in TNBC cells, mammosphere assay was conducted. Silencing of either BCL11A or DNMT1 resulted in decreased number of tumorospheres by over 50\% in both SUM149 and MDA-MB-231 cells (Fig. 6A). Since ISL1 was identified as a direct target of DNMT1, which was hypermethylated in breast cancer cells and BCSCs [22], we further checked the effect of BCL11A/DNMT1 on ISL1. As shown in Fig. 6B and 6C, consistently with previous study, ISL-1 was strongly suppressed in both normal cancer cells and BCSCs of SUM149 and MDA-MB-231. Knockdown of BCL11A and/or DNMT1 caused remarkable regains of ISL1 expression in both SUM149 and MDA-MB-231 cells. Similar results were also observed in SUM149 CSCs and MDA-MB-231 CSCs (Fig. 6B and 6C). Collectively, these data indicate that BCL11A/DNMT1 plays an important role in breast cancer stemness via suppressing ISL1 expression. 


\section{Cellular Physiology Cell Physiol Biochem 2018;47:2147-2158 \begin{tabular}{l|l} 
DOI: 10.1159/000491526 & $\begin{array}{l}\text { O 2018 The Author(s). Published by S. Karger AG, Basel } \\
\text { www.karger.com/cpb }\end{array}$ \\
\hline
\end{tabular}

\section{BCL11A-DNMT1 interaction is responsible for tumorigenesis in vivo}

In order to verify the regulatory effects of BCL11A and DNMT1 on tumor formation in vivo, BCL11A-knockdown or DNMT1-knockdown SUM149 and MDA-MB-231 cells were inoculated subcutaneously in nude mice and formation of tumors was monitored for 4 weeks. The tumors of either BCL11A-knockdown or DNMT1-knockdown cells were noticeably smaller than those of control cells (Fig. 7A). Silencing of BCL11A or DNMT1 in tumor tissues was confirmed by Q-PCR (Fig. 7B). Tumor volumes and weights of both BCL11A-knockdown and DNMT1-knockdown cells were remarkably lower than the control group (Fig. 7C and 7D). Taken together, these results suggest that shRNA-mediated disruption of BCL11ADNMT1 interaction could significantly impair tumor formation in vivo, indicating crucial roles of BCL11A-DNMT1 interaction in tumorigenesis of TNBC.

Fig. 6. BCL11A/DNMT1 is responsible for breast cancer stemness in vitro. A) Comparison of the number of suspension mammospheres formed from control cells, BCL11A knockdown cells and DNMT1 knockdown cells, both in SUM149 and MDA-MB-231 cells. Each bar is a mean $\pm \mathrm{SD}$ of $\mathrm{n}=$ 3 experiments. * indicates $\mathrm{P}<0.05$, ** indicates $\mathrm{P}<0.01$. B) ISL1 mRNA levels were determined by Q-PCR. GAPDH served as an internal control. Each bar is a mean \pm SD of $\mathrm{n}=3$ experiments. * indicates $\mathrm{P}<0.05$. C) ISL1 protein levels were determined by western blot. $\beta$-actin served as a loading control. Data are representative images of 3 independent experiments.
Fig. 7. BCL11A-DNMT1 interaction is responsible for tumorigenesis in vivo. A) Effect of BCL11A or DNMT1 knockdown on tumor formation in vivo. B) mRNA levels of BCL11A and DNMT1 in xenograft tumors were determined by Q-PCR. GAPDH served as an internal control. Each bar is a mean \pm SD of $n=3$ experiments. * indicates $\mathrm{P}<0.05$. C) Quantitative analysis of tumor volumes in BCL11A knockdown group or DNMT1 knockdown group. Each bar is a mean \pm SD of $\mathrm{n}=3$ experiments. $*$ indicates $\mathrm{P}<0.05$, ** indicates $\mathrm{P}<0.01$. D) Quantitative analysis of tumor weights in BCL11A knockdown group or DNMT1 knockdown group. Each bar is a mean $\pm \mathrm{SD}$ of $\mathrm{n}=3$ experiments. * indicates $\mathrm{P}<0.05$.

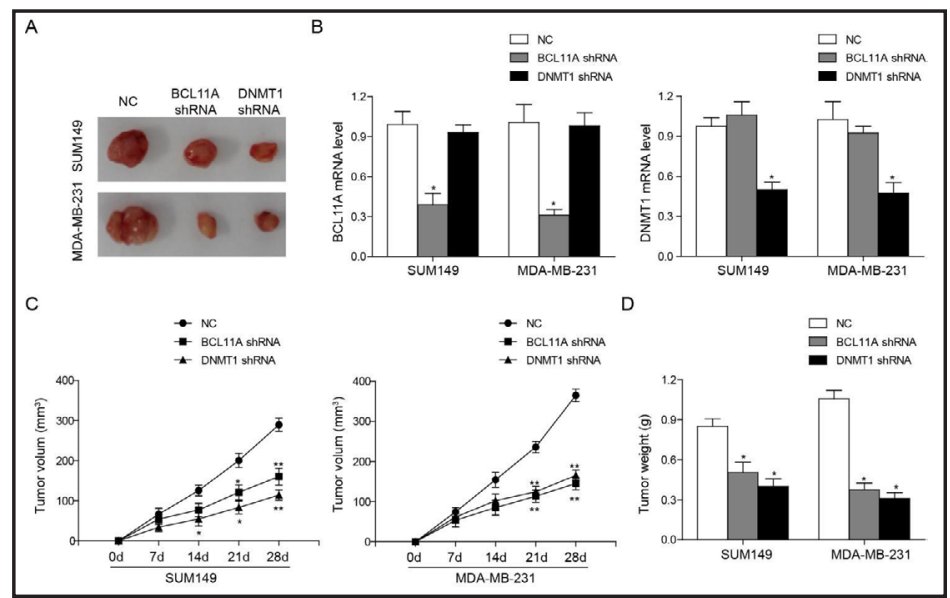

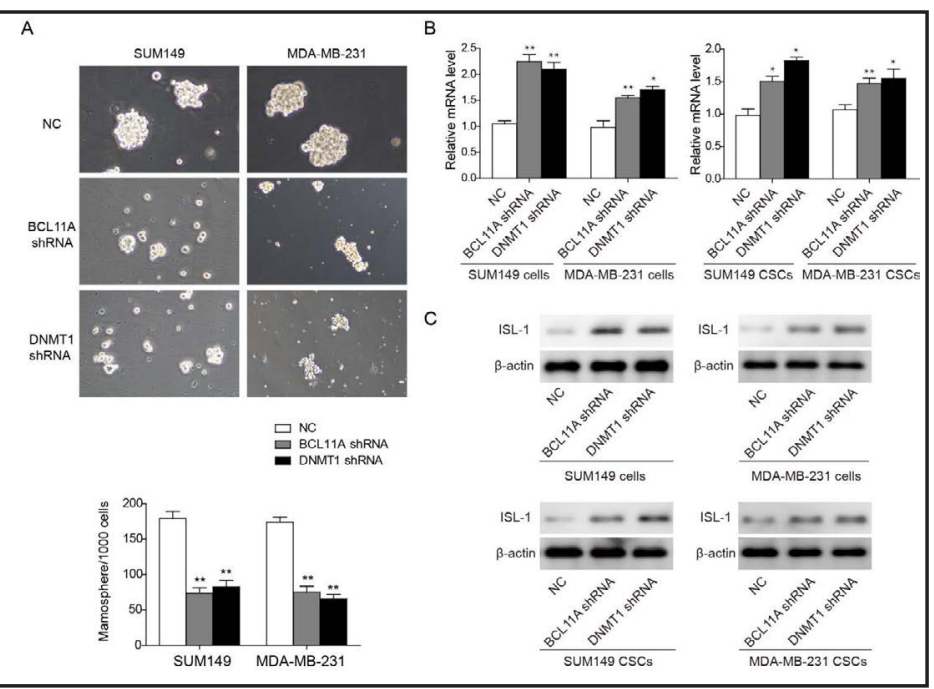




\section{Cellular Physiology Cell Physiol Biochem 2018;47:2147-2158

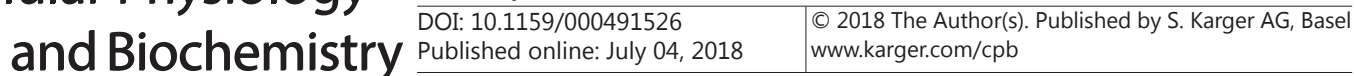 \\ Chen et al.: MiR-137 Impairs Cancer Stemness and Tumorigenesis in TNBC}

\section{Discussion}

BCL11A, a zinc-finger transcription factor, is well studied in hematopoiesis. In recent years, the clinical significance of BCL11A in solid tumors gained more and more attention. In current study, we have demonstrated that BCL11A is exclusively highly expressed in TNBC tissue and cell lines (Fig. 1B and 1D), which consistent with a recent report [20], suggesting that BCL11A might be a potential therapeutic target for TNBC. It will be important to identify the up- and down-stream molecular mechanisms of BCL11A in TNBC. miRNAs are crucial regulators of genes at post-transcriptional level. It's known that BCL11A is down-regulated by miR-30a in NSCLC cells [33], and modulated by miR-486-3p in human erythroid cells [34]. In this study, we have demonstrated for the first time that miR-137 acts as a tumor suppressor by targeting BCL11A in TNBC. Our findings suggest that miR-137-mediated down-regulation of BCL11A inhibits cancer stemness and tumor formation of TNBC cells in vitro and in vivo. This is consistent with an earlier report which showed that overexpression of miR-137 impairs tumor formation in TNBC cell lines [14, 16].

As shown in Fig. 2, miR-137 down-regulated BCL11A by directly targeting its 3' UTR. Furthermore, overexpression of miR-137 significantly decreased the population of BCSC and inhibited tumor development in vitro and in vivo. These effects were also observed in BCL11A-knockdown TNBC cells (Fig. 3 and 4). In addition, it has been reported that DNMT1 associates with BCL11A in primary erythroid cells, and it is required for maintenance of $\mathrm{HbF}$ silencing [25]. It is generally accepted that many tumor suppressor genes are suppressed by hypermethylation in their promoter region [21]. A novel carbazole SH-I-14-mediated disruption of STAT3-DNMT1 interaction leads to re-expression of tumor suppressor genes such as VHL and PDLIM4 via de-methylation in TNBC [26]. In our study, either BCL11A- or DNMT1 knockdown disrupted the interaction between these two proteins, which significantly impaired cancer stemness and suppressed tumor development in vitro and in vivo (Fig. 5, 6 and 7). Meanwhile, either miR-137 mimics or inhibitor showed few effects on DNMT1 expression (Fig. 5A and 5B), suggesting that miR-137 disrupts BCL11A-DNMT1 interaction via down-regulating BCL11A, and impairs cancer stemness and tumor development as a result.

More importantly, ISL1, a direct target of DNMT1, is hypermethylated and downregulated in a majority of breast cancer subtypes, including TNBC [22, 35]. Knockdown of DNMT1 or re-expression of ISL1 could strongly limit CSC population in breast cancer cells $[22,36]$. These observations thus prompted us to hypothesize that DNMT1 might cooperate with BCL11A to regulate cancer stemness and tumor development via hyper-methylating and suppressing ISL1 in TNBC. As expected, shRNA-mediated silencing of either BCL11A or DNMT1 led to a significant increase of ISL1 in both TNBC cells and TNBC CSCs (Fig. 6). These findings suggest that BCL11A/DNMT1 plays important roles in maintaining BCSC population, and contributes to breast cancer stemness and tumorigenesis by suppressing ISL-1 expression.

In conclusion, we demonstrated that in TNBC, miR-137 negatively regulates BCL11A expression by directly targeting its 3'UTR. MiR-137-mediated disruption of BCL11A-DNMT1 interaction impaired cancer stemness and tumorigenesis by suppressing ISL-1. Since BCSCs are responsible for chemoresistance and radioresistance [32]. Potential treatment targeting miR-137/BCL11A/DNMT1/ISL1 axis might increase the sensitive of TNBC cells to chemoand radiotherapy and improve the prognosis of TNBC.

\section{Acknowledgements}

We thank Professor Zhimin Shao (Department of Breast Surgery, Fudan University Shanghai Cancer Center) for the generous gift of SUM149 cell line. This work was supported by National Natural Science Foundation of China (No. 81302289).

\section{KARGER}




\section{Cellular Physiology Cell Physiol Biochem 2018;47:2147-2158

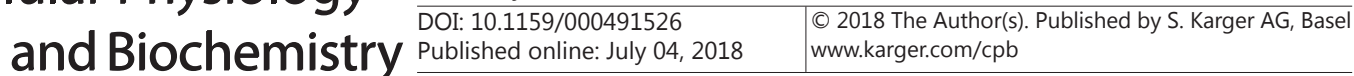

Chen et al.: MiR-137 Impairs Cancer Stemness and Tumorigenesis in TNBC

\section{Disclosure Statement}

The authors declare to have no competing interests.

\section{References}

-1 Goldhirsch A, Winer EP, Coates AS, Gelber RD, Piccart-Gebhart M, Thurlimann B, Senn HJ, Panel m: Personalizing the treatment of women with early breast cancer: highlights of the St Gallen International Expert Consensus on the Primary Therapy of Early Breast Cancer 2013 Ann Oncol 2013;24:2206-2223.

-2 Coates AS, Winer EP, Goldhirsch A, Gelber RD, Gnant M, Piccart-Gebhart M, Thurlimann B, Senn HJ, Panel M: Tailoring therapies--improving the management of early breast cancer: St Gallen International Expert Consensus on the Primary Therapy of Early Breast Cancer 2015 Ann Oncol 2015;26:1533-1546.

-3 Foulkes WD, Smith IE, Reis-Filho JS: Triple-negative breast cancer. N Engl J Med 2010;363:1938-1948.

4 Li J, Zhang BN, Fan JH, Pang Y, Zhang P, Wang SL, Zheng S, Zhang B, Yang HJ, Xie XM, Tang ZH, Li H, Li JY, He JJ, Qiao YL: A nation-wide multicenter 10-year (1999-2008) retrospective clinical epidemiological study of female breast cancer in China. BMC Cancer 2011;11:364.

-5 Idowu MO, Kmieciak M, Dumur C, Burton RS, Grimes MM, Powers CN, Manjili MH: CD44(+)/CD24(-/low) cancer stem/progenitor cells are more abundant in triple-negative invasive breast carcinoma phenotype and are associated with poor outcome. Hum Pathol 2012;43:364-373.

6 Mahamodhossen YA, Liu W, Rong-Rong Z: Triple-negative breast cancer: new perspectives for novel therapies. Med Oncol 2013;30:653.

7 Creighton CJ, Li X, Landis M, Dixon JM, Neumeister VM, Sjolund A, Rimm DL, Wong H, Rodriguez A, Herschkowitz JI, Fan C, Zhang X, He X, Pavlick A, Gutierrez MC, Renshaw L, Larionov AA, Faratian D, Hilsenbeck SG, Perou CM, Lewis MT, Rosen JM, Chang JC: Residual breast cancers after conventional therapy display mesenchymal as well as tumor-initiating features. Proc Natl Acad Sci U S A 2009;106:13820-13825. Li J, Li J, Wei T, Li J: Down-Regulation of MicroRNA-137 Improves High Glucose-Induced Oxidative Stress Injury in Human Umbilical Vein Endothelial Cells by Up-Regulation of AMPKalpha1 Cell Physiol Biochem 2016;39:847-859.

-9 Dyrskjot L, Ostenfeld MS, Bramsen JB, Silahtaroglu AN, Lamy P, Ramanathan R, Fristrup N, Jensen JL, Andersen CL, Zieger K, Kauppinen S, Ulhoi BP, Kjems J, Borre M, Orntoft TF: Genomic profiling of microRNAs in bladder cancer: miR-129 is associated with poor outcome and promotes cell death in vitro. Cancer Res 2009;69:4851-4860.

10 Zhang N, Wang X, Huo Q Sun M, Cai C, Liu Z, Hu G, Yang Q: MicroRNA-30a suppresses breast tumor growth and metastasis by targeting metadherin. Oncogene 2014;33:3119-3128.

11 Balaguer F, Link A, Lozano JJ, Cuatrecasas M, Nagasaka T, Boland CR, Goel A: Epigenetic silencing of miR137 is an early event in colorectal carcinogenesis. Cancer Res 2010;70:6609-6618.

12 Lin Y, Yang Y, Li W, Chen Q, Li J, Pan X, Zhou L, Liu C, Chen C, He J, Cao H, Yao H, Zheng L, Xu X, Xia Z, Ren J, Xiao L, Li L, Shen B, Zhou H, Wang YJ: Reciprocal regulation of Akt and Oct4 promotes the self-renewal and survival of embryonal carcinoma cells. Mol Cell 2012;48:627-640.

13 Zhao QW, Zhou YW, Li WX, Kang B, Zhang XQ Yang Y, Cheng J, Yin SY, Tong Y, He JQ, Yao HP, Zheng M, Wang YJ: Aktmediated phosphorylation of Oct4 is associated with the proliferation of stemlike cancer cells. Oncol Rep 2015;33:1621-1629.

14 Zhao Y, Li Y, Lou G, Zhao L, Xu Z, Zhang Y, He F: MiR-137 targets estrogen-related receptor alpha and impairs the proliferative and migratory capacity of breast cancer cells. PLoS One 2012;7:e39102.

15 Han Y, Bi Y, Bi H, Diao C, Zhang G, Cheng K, Yang Z: miR-137 suppresses the invasion and procedure of EMT of human breast cancer cell line MCF-7 through targeting CtBP1 Hum Cell 2016;29:30-36.

16 Lee JM, Cho KW, Kim EJ, Tang Q, Kim KS, Tickle C, Jung HS: A contrasting function for miR-137 in embryonic mammogenesis and adult breast carcinogenesis. Oncotarget 2015;6:22048-22059.

17 Bauer DE, Orkin SH: Hemoglobin switching's surprise: the versatile transcription factor BCL11A is a master repressor of fetal hemoglobin. Curr Opin Genet Dev 2015;33:62-70.

18 Nakamura T, Yamazaki Y, Saiki Y, Moriyama M, Largaespada DA, Jenkins NA, Copeland NG: Evi9 encodes a novel zinc finger protein that physically interacts with BCL6, a known human B-cell proto-oncogene product. Mol Cell Biol 2000;20:3178-3186. 


\section{Cellular Physiology Cell Physiol Biochem 2018;47:2147-2158 \begin{tabular}{l|l} 
DOI: 10.1159/000491526 & $\begin{array}{l}\text { O 2018 The Author(s). Published by S. Karger AG, Basel } \\
\text { www.karger.com/cpb }\end{array}$ \\
\hline
\end{tabular}}

19 Satterwhite E, Sonoki T, Willis TG, Harder L, Nowak R, Arriola EL, Liu H, Price HP, Gesk S, Steinemann D, Schlegelberger B, Oscier DG, Siebert R, Tucker PW, Dyer MJ: The BCL11 gene family: involvement of BCL11A in lymphoid malignancies. Blood 2001;98:3413-3420.

20 Khaled WT, Choon Lee S, Stingl J, Chen X, Raza Ali H, Rueda OM, Hadi F, Wang J, Yu Y, Chin SF, Stratton M, Futreal A, Jenkins NA, Aparicio S, Copeland NG, Watson CJ, Caldas C, Liu P: BCL11A is a triple-negative breast cancer gene with critical functions in stem and progenitor cells. Nat Commun 2015;6:5987.

21 Agrawal A, Murphy RF, Agrawal DK: DNA methylation in breast and colorectal cancers. Mod Pathol 2007;20:711-721.

-22 Pathania R, Ramachandran S, Elangovan S, Padia R, Yang P, Cinghu S, Veeranan-Karmegam R, Arjunan P, Gnana-Prakasam JP, Sadanand F, Pei L, Chang CS, Choi JH, Shi H, Manicassamy S, Prasad PD, Sharma S, Ganapathy V, Jothi R, Thangaraju M: DNMT1 is essential for mammary and cancer stem cell maintenance and tumorigenesis. Nat Commun 2015;6:6910.

23 Lee E, Wang J, Yumoto K, Jung Y, Cackowski FC, Decker AM, Li Y, Franceschi RT, Pienta KJ, Taichman RS: DNMT1 Regulates Epithelial-Mesenchymal Transition and Cancer Stem Cells, Which Promotes Prostate Cancer Metastasis. Neoplasia 2016;18:553-566.

-24 Zagorac S, Alcala S, Fernandez Bayon G, Bou Kheir T, Schoenhals M, Gonzalez-Neira A, Fernandez Fraga M, Aicher A, Heeschen C, Sainz B, Jr: DNMT1 Inhibition Reprograms Pancreatic Cancer Stem Cells via Upregulation of the miR-17-92 Cluster. Cancer Res 2016;76:4546-4558.

-25 Xu J, Bauer DE, Kerenyi MA, Vo TD, Hou S, Hsu YJ, Yao H, Trowbridge JJ, Mandel G, Orkin SH: Corepressordependent silencing of fetal hemoglobin expression by BCL11A. Proc Natl Acad Sci U S A 2013;110:65186523.

-26 Kang HJ, Yi YW, Hou SJ, Kim HJ, Kong Y, Bae I, Brown ML: Disruption of STAT3-DNMT1 interaction by SH-I14 induces re-expression of tumor suppressor genes and inhibits growth of triple-negative breast tumor. Oncotarget 2015;10.18632/oncotarget.4054

-27 Borcherding N, Kusner D, Kolb R, Xie Q, Li W, Yuan F, Velez G, Askeland R, Weigel RJ, Zhang W: Paracrine WNT5A Signaling Inhibits Expansion of Tumor-Initiating Cells. Cancer Res 2015;75:1972-1982.

28 Jin K, Xiang Y, Tang J, Wu G, Li J, Xiao H, Li C, Chen Y, Zhao J: miR-34 is associated with poor prognosis of patients with gallbladder cancer through regulating telomere length in tumor stem cells. Tumour Biol 2014;35:1503-1510.

29 Lin X, Liu S, Luo X, Ma X, Guo L, Li L, Li Z, Tao Y, Cao Y: EBV-encoded LMP1 regulates Op18/stathmin signaling pathway by cdc2 mediation in nasopharyngeal carcinoma cells. Int J Cancer 2009;124:10201027.

-30 Lee HE, Kim JH, Kim YJ, Choi SY, Kim SW, Kang E, Chung IY, Kim IA, Kim EJ, Choi Y, Ryu HS, Park SY: An increase in cancer stem cell population after primary systemic therapy is a poor prognostic factor in breast cancer. Br J Cancer 2011;104:1730-1738.

31 Liu C, Luo Y, Liu X, Lu P, Zhao Z: Clinical implications of CD44+/CD24- tumor cell ratio in breast cancer. Cancer Biother Radiopharm 2012;27:324-328.

-32 Carrasco E, Alvarez PJ, Prados J, Melguizo C, Rama AR, Aranega A, Rodriguez-Serrano F: Cancer stem cells and their implication in breast cancer. Eur J Clin Invest 2014;44:678-687.

-33 Jiang BY, Zhang XC, Su J, Meng W, Yang XN, Yang JJ, Zhou Q, Chen ZY, Chen ZH, Xie Z, Chen SL, Wu YL: BCL11A overexpression predicts survival and relapse in non-small cell lung cancer and is modulated by microRNA-30a and gene amplification. Mol Cancer 2013;12:61.

-34 Lulli V, Romania P, Morsilli O, Cianciulli P, Gabbianelli M, Testa U, Giuliani A, Marziali G: MicroRNA-486-3p regulates gamma-globin expression in human erythroid cells by directly modulating BCL11A. PLoS One 2013;8:e60436.

-35 Gay F, Anglade I, Gong Z, Salbert G: The LIM/homeodomain protein islet-1 modulates estrogen receptor functions. Mol Endocrinol 2000;14:1627-1648.

36 Morita R, Hirohashi Y, Suzuki H, Takahashi A, Tamura Y, Kanaseki T, Asanuma H, Inoda S, Kondo T, Hashino S, Hasegawa T, Tokino T, Toyota M, Asaka M, Torigoe T, Sato N: DNA methyltransferase 1 is essential for initiation of the colon cancers. Exp Mol Pathol 2013;94:322-329. 